\title{
Protecting Japan from influenza
}

To the editor-Kennedy F. Shortridge's News and Views article in the April issue of Nature Medicine ${ }^{1}$ recalls that influenza has been a significant and highly contagious disease for a long time. It has been estimated that the infamous 1918 epidemic killed about 700,000 Japanese in just a few months. Today, influenza can still lead to death, especially in infants and the elderly, and Japan is seeing too many people succumb to this disease. The fact that most of the deceased have not been

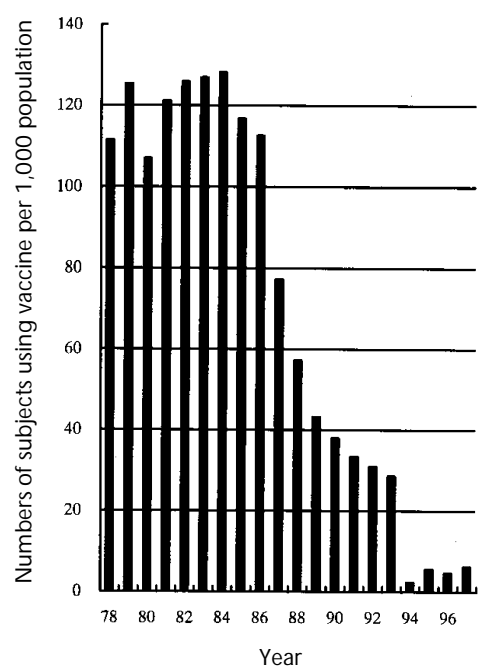

\section{Cytokine modulatio}

To the editor-In the March issue of Nature Medicine, Grivel and Margolis ${ }^{1}$ presented work on the cytopathicity of CCR5-and CXCR4-tropic HIV-1, concluding that, contrary to earlier thinking, virus using the CCR5 receptor to gain entry to T cells (R5 isolates) are as cytopathic as virus using the CXCR4 receptor (X4 isolates). They went on to suggest that the noted lower pathogenicity of R5 is due to lower numbers of cells bearing the CCR5 receptor in the target tissue, in this case tonsil explants. However, the authors used tonsil tissue from HIV-seronegative individuals undergoing tonsillectomy. This tissue may not have the same distribution of CCR5 and CXCR4 receptors as that of HIV-infected patients.

We have recently demonstrated a significant upregulation of CCR5 and CXCR4 receptors (five- to tenfold, or $30-40 \%$ of all CD 4 cells) in tonsillar tissue obtained from asymptomatic anti-retroviral-naive, HIV-1-infected individuals (with peripheral blood CD4 counts of more than 500 cells/ $\mu \mathrm{l}$ ) compared with vaccinated suggests the need to reevaluate the prophylactic measures that exist for managing influenza in Japan.

The rate of influenza vaccination has been declining in Japan (see Fig.). In the early 1980s, the rate of vaccination in school children was about $80 \%$. By 1992 , this figure had dropped to $18 \%$ (ref. 2). Moreover, a 1994 amendment of the prophylactic vaccination law which made it no longer compulsory to receive this vaccine, has resulted in a further drop to lessthan $10 \%$. The sametrend can be seen among elderly patients.

The drop in vaccination rates has been accompanied by skepticism about the effectiveness of prophylactic vaccination for influenza, in part because of poorly designed studies ${ }^{3}$. As a result, the annual production of vaccine, which peaked at 26,216 liters in 1967, dropped to only 596 liters in 1996.

Fig. 1 Number of subjects using influenza vaccine per 1,000 people in Japan between 1978 and 1997. The pre-1994 values were calculated from the numbers of subjects who received influenza vaccinations twice, which has been the formal method in Japan. The post-1994 values were estimated from the numbers of doses produced per 1,000 population because information on the actual vaccination was not available.
To promote anti-influenza vaccinations, the health authorities seem to be considering revision of the wording from "arbitrary vaccination" to "official recommendation of vaccination" when the prophylactic vaccination law is reviewed next fiscal year ${ }^{4}$. Japan's system for the mass production of vaccine must be reviewed accordingly. The dramatic decline in vaccine demand has almost eliminated domestic farm production of hen eggs for vaccine production. To restore social protection against influenza, the nation's authorities will have to rebuild the vaccine mass production system, in addition to obtaining the public trust on the efficacy of vaccination.

\section{KAZUO INOUE}

Towa Clinic

Dept. of Community and Family M edicine Jichi Medical School

468 Showa, Towa, Hata

Kochi 786-0511 Japan

1. Shortridge, K.F. The 1918 'Spanish' flu: pearls from swine? Nature Med. 5, 384-385 (1999).

2. Hirota, Y., Fedson, D.S. \& Kaji, M. Japan lagging in influenza jabs. Nature 380, 18 (1996).

3. Hirota, Y. \& Kaji, M. Scepticism about influenza vaccine efficacy in Japan. Lancet 344, 408-409 (1994).

4. "Influenza saizensen (The front line of influenza)" in Kochi Shimbun Newspaper 13, 28 January 1999.

\section{of HIV-1 chemokine receptor expression}

that of HIV-negative control tissue from patients with tonsillar hypertrophy or recurrent tonsillitis ${ }^{2}$. This upregulation of CCR5 in tonsil tissue correlated with high levels of IL2 and $\gamma$-interferon expression. We have also shown that in vitro these type 1 cytokines can upregulate CCR5 as well as CXCR4 receptor expression on CD4 cells.

We therefore believe that the authors' interpretation of their data may be overly simplistic and that a more complex interaction between cytokines and chemokine receptor exist in vivo in HIV-infected lymphoid sites. This immune reaction in vivo may protect CD4 cells from HIV chemokine receptor-mediated cell death, noted in the explant model, and generate huge numbers of HIV-susceptible CD4 cells in the lymphoid tissue.

Alan L. Landay ${ }^{1}$, Bruce Patterson ${ }^{2}$ $\&$ JAN ANDERSSON ${ }^{3}$

${ }^{1}$ D epartment of Immunology/M icrobiology Rush Medical College, Chicago, Illinois USA ${ }^{2}$ D ept. of Obstetrics/Gynecology and M edicine
Division of Infectious Disease

N orthwestern University M edical School

Chicago, Illinois, USA

${ }^{3}$ Division of Infectious Diseases, 163

D epartment of Immunology/M icrobiology

Pathology and infectious Diseases

Karolinska Institute

Huddinge University Hospital

S-141 85 Huddinge, Sweden

Margolis and Grivel reply_-In our paper we found that in human tonsillar tissue ex vivo the majority of CD4 cells express CXCR4, whereas a minority express CCR5. We demonstrated that R5 HIV-1 variants deplete only CCR5-expressing CD4 cells. This explainswhy, in spite of a high cytopathicity for their cognatetarget cells, R5 variants deplete CD4 cells less than X4 variants do.

Both in vivo and ex vivo lymphocyte expression of co-receptor can be modulated by various factors ${ }^{3}$ including cytokines, as indicated by Landay et al. In chronically HIV-infected individuals, immune activation may thus modulate CCR5 and/or CXCR4 co-receptor expression ${ }^{2}$ and there- 